\title{
¿POR QUÉ AUDITAR A LOS COMITÉS DE ÉTICA EN INVESTIGACIÓN?
}

\author{
Estela Quiroz ${ }^{1}$
}

\begin{abstract}
RESUMEN
Los comités de ética en investigación biomédica tienen como responsabilidad asegurar la protección de los participantes humanos en las investigaciones. Para mejorar la calidad de su trabajo deben someterse a procedimientos de auditoría, encargadas por los patrocinadores, y de inspecciones por parte de la autoridad regulatoria. A través de dichos procedimientos se garantizaría que mejoren sus funciones, y así puedan perfeccionar su labor y cumplir de manera óptima con el objetivo para el que fueron creados.
\end{abstract}

Palabras clave: Comités de Ética; Auditoría administrativa; Control de calidad; Ensayos clínicos como asunto; Perú (fuente: DeCS BIREME).

\section{WHY TO AUDIT TO RESEARCH ETHICS COMMITTEES?}

\begin{abstract}
Ethics committees in biomedical research have the responsibility to ensure the protection of human participants in the studies. In order to improve the quality of their work they must undergo audit procedures commissioned by the sponsors and inspections done by the regulatory authorities. Through these procedures, improvement of their functions should be guaranteed, so they can optimize their tasks and accomplish in the best way the purpose for which they were created.
\end{abstract}

Key words: Ethics Committees; Management audit; Quality control; Clinical trials as topic; Peru (source: MeSH NLM).

\section{INTRODUCCIÓN}

Los comités institucionales de ética en investigación biomédica (CEI), son definidos como organizaciones independientes (consejo de revisión, comité institucional, regional, nacional o supranacional), integrados por profesionales médicos científicos y miembros no médicos/no científicos ${ }^{(1)}$.

Su responsabilidad es asegurar la protección de los derechos, la seguridad y el bienestar de los seres humanos involucrados en una investigación, y proporcionar una garantía pública de esa protección, a través, entre otras cosas, de la revisión y aprobación/ opinión favorable del protocolo de estudio, de la capacidad del/los investigador/es y lo adecuado de las instalaciones, de los métodos y materiales que se usarán, al obtener y documentar el consentimiento informado de los sujetos del estudio. El estado legal, la composición, función, los requerimientos regulatorios y de operación de los CEI pueden diferir entre los países, pero deben permitir que el CEI actúe de acuerdo con las Buenas Prácticas Clínicas (BPC) ${ }^{(1)}$.
Sin embargo la realidad muestra que no siempre los comités pueden cumplir con todo lo que se espera de ellos, debido a muchas causas. Entre las más importantes figuran:

- Carencia de los recursos mínimos necesarios, muchos funcionan en oficinas pequeñas, escasamente acondicionadas ${ }^{(2)}$;

- Carencia del equipamiento para archivar los numerosos documentos que reciben (los que además de ser archivados adecuadamente, deben tener garantizada la confidencialidad) (3);

- Sus miembros a menudo dedican al comité un tiempo adicional al trabajo diario que realizan en las instituciones a las que pertenecen ${ }^{(3)}$;

- Muchos no cuentan con la capacitación suficiente en los aspectos relacionados con la ética de la investigación ${ }^{(2,3)}$, a pesar de la labor que realiza la Red de Comités de Ética en Investigación (4), el Instituto de Ética de la Universidad Nacional Mayor de San Marcos y el Instituto Nacional de Salud como instancia encargada de la regulación de los ensayos clínicos en el Perú;

\footnotetext{
Médica oftalmóloga, docente de ética y metodología de la investigación, Coordinadora de la Red Peruana de Comités de Ética de la Investigación. Hospital Nacional Hipólito Unanue. Lima, Perú.
}

Recibido: 06-07-10 Aprobado: 11-08-10 
- Muchas veces no existe ninguna compensación por el trabajo que realizan, lo que desanima o hace que los miembros externos falten a menudo a las reuniones ${ }^{(2,3)}$;

- Sufren la presión de más trabajo, cada día es mayor el número de protocolos nuevos que ingresan para revisión; además, deben lidiar con un gran número de reportes de eventos adversos, cartas de los investigadores, solicitudes de aprobación de enmiendas, revisión de nuevas versiones de consentimientos, etc. ${ }^{(5)}$;

- Muchos no cuentan al menos con una secretaria que se encargue de las labores administrativas, por no mencionar que casi ninguno cuenta con un administrador que coordine las actividades ${ }^{(2)}$;

- Muchos comités tienen reglamento, pero no cuentan con procedimientos operativos estandarizados (POE: instrucciones detalladas escritas, para obtener uniformidad en la realización de una función específica) ${ }^{(6,7)}$ propios, a pesar de que la guía de BPC de la Conferencia Internacional de Armonización (CIARM) es muy clara en recomendar de que los CEI deberían contar con ellos ${ }^{(8)}$.

EI CEI cada día tiene mayores responsabilidades, ya que además de la consulta ética, educación y revisión por pares que realiza, se le solicita que cumpla una función más directa en la revisión de los conflictos financieros y de interés que pudieran presentarse o que haga seguimiento de los estudios aprobados ${ }^{(9)}$, lo cual rara vez logra hacer, y todo esto en el ambiente cada día más complejo de la investigación biomédica.

Todo lo anterior es más notorio en los países en desarrollo, pues en ellos muchos comités surgieron como una necesidad, cuando las investigaciones multicéntricas, en su mayoría patrocinadas por la industria farmacéutica, se empezaron a realizar en instituciones locales, para las cuales las recomendaciones (CIOMS Pauta 3$)^{(10)}$ y la reglamentación internacional (11) requieren la aprobación de los protocolos por un comité local, lo que también figura como requisito en la regulación peruana que norma la realización de los ensayos clínicos ${ }^{(12)}$.

A esto se suma el hecho de que en muchos países de la región no existe aún el marco legal necesario que establezca políticas claras para normar la actividad de los comités o que, cuando están definidos en la reglamentación de los ensayos clínicos, su definición era ambigua ${ }^{(13,14)}$. En consecuencia, vemos que para constituirse y funcionar, los comités deberían seguir las recomendaciones estipuladas tanto en las regulaciones internacionales, como el Código de Reglamentos Federales, Título 45, Sección $46.103^{(11)}$ así como en los diversos documentos elaborados al respecto (Guía de Buenas Prácticas Clínicas: E6 ${ }^{(8)}$, Guías europeas para auditar comités ${ }^{(15,16)}$, o las guías de la Organización Mundial de la Salud ${ }^{(6,10)}$, e incluso modelos preparados de POE) (7). Sin embargo, no todos cumplen los requisitos mínimos establecidos, por lo que en la realidad el fortalecimiento de los CEI es una necesidad, ya que funcionan como pueden, y aún no está claro cual debería ser el organismo encargado de acreditarlos, auditarlos o inspeccionarlos, para que así se garantice que sigan procedimientos operativos estándar y el trabajo que realizan sea cumplido a cabalidad.

Fuentes \& Revilla (3) concluyen estableciendo La necesidad de implementar la acreditación de los CEI, particularmente en el Perú, como parte de un proceso que sea factible y adecuado según nuestra propia realidad, del mismo modo Minaya-Martínez \& Diaz-Sandoval (17), al referirse a la capacidad de evaluación de los ensayos clínicos por los CEl, sugieren Promover la constitución de una Comisión Nacional de Ética en Investigación que registre, acredite y monitorice a los CEI.

El objetivo del presente ensayo es plantear la necesidad e importancia de que los comités que evalúan los aspectos éticos de las investigaciones, sean sujetos a evaluación dentro de un sistema de gestión de calidad, debido a la gran responsabilidad que tienen con la sociedad y, en especial, con los participantes en las investigaciones.

\section{SISTEMA DE GESTIÓN DE CALIDAD}

A nivel empresarial, se define al sistema de gestión de calidad como una opción para optimizar recursos y lograr la satisfacción del cliente ${ }^{(18)}$, según la Food and Drug Administration (FDA) de EEUU, dicha definición aplicada a la investigación biomédica se refiere a: Las prácticas formalizadas que definen las responsabilidades del gerenciamiento, procesos, procedimientos y recursos necesarios para cumplir con los requerimientos del producto o servicio, la satisfacción del cliente y la mejora continua de la calidad ${ }^{(a)}$. Esta es una definición modificada de la definición previamente establecida por el European Forum Good Clinical Practices (EFGCP) que si bien era similar, es un poco menos específica.

Basados en las intenciones de las BPC ${ }^{(8)}$, que son definidas como: Un estándar internacional de calidad

\footnotetext{
a Mejora continua de la calidad. Actividad recurrente para aumentar la capacidad de cumplir con los requisitos solicitados a través del esfuerzo para evaluar los niveles de funcionamiento y ejecución de las tareas en un programa o sitio (18).
} 
ética y científica para diseñar, conducir, archivar $y$ reportar ensayos con la participación de seres humanos, y que la adherencia a estas provee la seguridad sobre la precisión e integridad de los datos del estudio así como sobre la protección de los sujetos participantes, surge la necesidad de la implementación de un sistema de gestión de calidad, en el ámbito de la investigación clínica.

Todo sistema de gestión de calidad se basa en dos aspectos fundamentales: aseguramiento de la calidad (b) y control de la calidad (c).

A pesar de que ni la FDA ni la Conferencia Internacional de Armonización establecen como mandatorio los programas de aseguramiento de la calidad, en el lugar donde se realizan las investigaciones, se hace mucho énfasis en el cumplimiento de la Guía de Buenas Prácticas de Manufactura (GMP), o de Laboratorio y Tecnología (GLP) ${ }^{(19)} \mathrm{y}$, por supuesto, de las Buenas Prácticas Clínicas (BPC).

En cuanto a las mecanismos para objetivar y, de algún modo, asegurar la adherencia a estas buenas prácticas, existen procesos definidos claramente en el glosario de la Conferencia Internacional de Armonización ${ }^{(8)}$, tales como:

Monitoreo. Acto de vigilar el proceso de un estudio clínico y asegurarse de que este sea conducido, registrado y reportado de acuerdo con el protocolo, POE y las BPC y los requerimientos regulatorios aplicables.

Auditoría. Examen sistemático e independiente de las actividades y documentos relacionados con el estudio para determinar si las actividades evaluadas fueron realizadas y los datos fueron registrados, analizados y reportados con exactitud de acuerdo con el protocolo, los POE del patrocinador, las BPC, y los requerimientos regulatorios aplicables.

Ambos procedimientos, por definición, son muy similares, ambos son responsabilidad del Patrocinador, quien a través de ellos garantiza un sistema de aseguramiento y mejora continua de la calidad. En el caso de que en el monitoreo se detecte alguna falla en los procesos, de inmediato se puede implementar acciones para corregir cualquier cosa que pudiera causar algún perjuicio a los sujetos que participan en las investigaciones; del mismo modo, cualquier situación que pudiera atentar contra la calidad de los datos será corregida de inmediato y así evitar "desastres" como datos fraudulentos, por ejemplo.

En el caso de la auditoría, (realizada por personal de la empresa o instituciones especializadas contratadas por el patrocinador), el informe podría conducir al cierre de un sitio de investigación, al demostrar que, a pesar de las recomendaciones recibidas en el proceso de monitoreo, continúa la falta de adherencia a las BPC, y se siguen poniendo en riesgo la calidad de los datos (objetivo principal del patrocinador) así como la seguridad de los participantes en la investigación.

Por otro lado, la auditoría como parte del proceso de aseguramiento de la calidad y como una necesidad de proteger a los sujetos humanos que participan en las investigaciones, es similar a las inspecciones en cuanto al procedimiento en sí.

Inspección. Se define como la Acción de las autoridades regulatorias de realizar una revisión oficial de los documentos, instalaciones, registro y de cualquier otro recurso que las autoridades consideren esté relacionado con el estudio clínico y que pueda ser localizado en el sitio donde se realiza el estudio, en las instalaciones del patrocinador o de la organización de la investigación por contrato (OIC) o en otros sitios que las autoridades regulatorias consideren apropiados ${ }^{(8)}$.

La principal diferencia con las auditorías, como se puede notar en la definición, es que las inspecciones son desarrolladas por la autoridad regulatoria, además, que se realizan generalmente al terminar los estudios, salvo que durante la realización de estos haya una denuncia ante la sospecha de que pudieran estar violándose los derechos o la seguridad de los participantes.

Prácticamente hablando, las agencias regulatorias, los patrocinadores y las OIC -que los representan muchas veces-, tienen un gran poder pues pueden cerrar a la entidad inspeccionada/auditada; $y$, en el caso de las inspecciones después de terminados los estudios, rechazar los estudios propuestos a la FDA, por ejemplo ${ }^{(20)}$.

Los principios de aseguramiento de la calidad/control de calidad, especialmente aquellos que enfatizan el "mejoramiento continuo", sugieren que una auditoría revela fortalezas y debilidades de los procedimientos operativos. Tradicionalmente, la auditoría se enfoca

\footnotetext{
b Aseguramiento de la calidad. Son todas aquellas acciones planeadas y sistemáticas que se establecen para garantizar que el estudio se está realizando y que los datos son generados, documentados (registrados) y reportados en cumplimiento con la BPC y los requerimientos regulatorios aplicables ${ }^{(8)}$.

c Control de la calidad. Las técnicas y actividades operacionales realizadas dentro del sistema de aseguramiento de la calidad para verificar que se han cumplido los requerimientos de calidad de las actividades relacionadas con el estudio ${ }^{(8)}$.
} 
en la consistencia de la documentación y en la reproducibilidad de un evento o serie de eventos, vía la documentación. Sin embargo, el enfoque más reciente está relacionado con que no deben ser procedimientos fríos e inconsistentes, sino más interactivos, cuyos resultados deben ser comunicados no solo a quien los solicitó sino a los auditados (investigadores, comités de ética, u otros), para de ese modo contribuir a la comprensión de los motivos por los que no se adhirieron a las BPC, y corregir las actividades deficientes ${ }^{(21)}$.

\section{Y, ¿LOS COMITÉS DE ÉTICA?}

Con relación a la Guía de Buenas Prácticas Clínicas y a los procesos de auditoría interna de los comités de ética, existen pocos reportes y se refieren a las auditorías llevadas a cabo por compañías externas que determinan si los comités se adhieren a los requerimientos regulatorios federales en EEUU ${ }^{(22)}$, muchas de ellas realizadas a solicitud de la Oficina de Protección para la Investigación con Seres Humanos del Departamento de Salud y Servicios Humanos y de la FDA, generalmente, en respuesta a los cada vez más publicitados casos enfocados a la incapacidad de los comités de proteger a los participantes en las investigaciones, ejemplo de ello son los conocidos casos de Jesse Gelsinger y Ellen Roche ${ }^{(23)}$, ambos voluntarios en investigaciones que fueron aprobadas por los respectivos comités, los cuales, después que estos participantes fallecieran, fueron inspeccionados y sancionados. Peor aun es el caso de denuncias periodísticas directas a las agencias regulatorias, que se supone son las responsables de las inspecciones, que hacen una clara y directa alusión a la participación de los comités en presuntas irregularidades dentro del marco de la investigación clínica ${ }^{(24)}$.

De allí que es importante hacer hincapié en la necesidad de garantizar la adherencia y el aseguramiento de la calidad -la adherencia de todos los implicados en la investigaciónno solo los investigadores, y el aseguramiento de todas las entidades comprometidas en la investigación clínica: administrativas, legales, regulatorias, bioseguridad, farmacia, enfermería, manejo de la calidad y el riesgo y, por supuesto de los comités de ética.

Por otro lado, la protección de los sujetos de investigación es tan importante que no se la puede dejar a cargo de los CIE solamente. La idea es que todos los actores involucrados en la investigación (desde la autoridad regulatoria, pasando por los patrocinadores, organizaciones de investigación por contrato, sitios de investigación, CIE, instituciones académicas, centros médicos, hasta las organizaciones de la comunidad) compartan una responsabilidad básica, esta es, proteger el bienestar y los derechos de los individuos que aceptan participar voluntariamente en los ensayos clínicos. Lo cual constituye una visión más formal de la relación entre la adherencia (incluyendo las auditorías) a las BPC y las culturas dentro de las cuales se está llevando a cabo la investigación.

Así es que, si se considera a los CIE dentro de este sistema, estos deberían cumplir con las exigencias requeridas para un óptimo funcionamiento cuyo producto final garantice la satisfacción de sus clientes: los sujetos participantes en las investigaciones.

En la actualidad hay patrocinadores que consideran auditar a los CIE a pesar de lo que esto implica, en relación con la confidencialidad de los resultados de dichas auditorías o a la conducta que debieran tomar si se encontrara que estos no funcionan adecuadamente, así como la falla en apreciar las interacciones entre el sitio y el comité entre otros posibles hallazgos; los ¿qué?, ¿cuándo?, y ¿por qué?, que comprometen el valor y la integridad de una auditoría ${ }^{(20)}$.

Así, enfrentados al reto de desarrollar e implementar terapias novedosas rápidamente, existe una necesidad seria de considerar las políticas, prácticas y regulaciones públicas, e institucionales que contribuyan al desarrollo adecuado de $\mathrm{CEI}$, altamente profesionales y adecuadamente implementados, e incluso considerar en las normas la posibilidad de que sean financiados por la industria ${ }^{(25)}$ a través de entidades que no comprometan su independencia, de tal manera que los servicios de los integrantes del comité, que hasta ahora en muchos casos siguen siendo gratuitos, puedan ser reconocidos adecuadamente.

Una vez garantizada la logística apropiada para su funcionamiento y las posibilidades de cumplir con las funciones educativas de sus miembros, deberían no solo ser sometidos a procesos de monitoreo interno, o de auditoría sino que deberían ser inspeccionados por entidades ajenas a ellos, siguiendo las recomendaciones de la Organización Mundial de la Salud, quienes sin decir a quien le compete esta tarea hablan del surveyor $y$, establecen claramente los mecanismos de control para los CEI (6).

En nuestros países y asociado al factor cultural referido a "cumplir por temor a las sanciones", a la par de preocuparnos por qué la normativa al respecto sea aprobada lo antes posible, se debería empezar un proceso de inspección a todos los comités, con carácter diagnósticoal inicio, para progresivamente irimplementado el proceso de mejoramiento de la calidad, para el cual los comités deberían tender a lograr la acreditación, 
proceso por el cual un organismo autorizado reconoce formalmente que una organización es competente para la realización de una determinada actividad de evaluación de la conformidad de su trabajo.

Se dice que la acreditación es un medio de suplantar o al menos reducir la necesidad de auditar ${ }^{(26)}$. Dependiendo de cómo la estructura de la acreditación esté instaurada, particularmente quienes se constituyen en los revisores frente a los auditores de la entidad de acreditación, ello podría evitar otro problema para los auditores: ¿hasta dónde puede un grupo que realiza una auditoría, compartir información con otros de la misma entidad?

Puesto en concreto: ¿qué tanto puede o podría un patrocinador compartir información de la auditoría que hizo a un comité, por ejemplo con otros patrocinadores u otros comités?

La acreditación se basa en estándares que se derivan de las regulaciones, guías y mejores prácticas. Las entidades formales e independientes que se encargan de acreditar dan la conformidad a aquellos que solicitan voluntariamente someterse a una acreditación; mientras las regulaciones y las guías se constituyen en el piso, o el mínimo, la acreditación intenta codificar más que la actividad mínima aceptable de la entidad acreditada para que esta logre la mejor práctica posible.

Lo que constituiría un cambio en los objetivos, ir de una cultura de adherencia a una cultura de conciencia. Las implicancias son importantes. La cultura de adherencia es manejada por las regulaciones y la cultura de conciencia es manejada por una cultura de responsabilidad y compromiso profesional compartido. La implementación no es fácil, es sistemática y lenta, pero además necesaria ${ }^{(27)}$.

En conclusión, las auditorías o las inspecciones dentro del marco de la acreditación, constituyen mecanismos importantes en el proceso de mejorar la calidad de los Comités de Ética en Investigación en aras de conseguir su objetivo, brindar la mejor protección a los seres humanos que participan en las investigaciones.

\section{AGRADECIMIENTOS}

A la Dra. Florencia Luna por su apoyo en la revisión del presente ensayo. Al Dr. Percy Mayta-Tristán por el estímulo que me brindó para su publicación.

\section{Conflictos de interés}

La autora declara no tener conflictos de interés en la publicación de este artículo.

\section{REFERENCIAS BIBLIOGRÁFICAS}

1. Organización Panamericana de la Salud. Buenas prácticas clínicas: documento de las Américas. Washington DC: OPS; 2005.

2. Lecca L, Llanos-Zavalaga F, Ygnacio E. Características de los comités de ética en investigación del Perú autorizados para aprobar ensayos clínicos. Rev Med Hered. 2005;16(1):3-10.

3. Fuentes D, Revilla D. Acreditación de comités de ética en investigación, como parte de un proceso. An Fac Med (Lima). 2007;68(1):67-74.

4. Lescano AR, Blazes DL, Montano SM, Moran Z, Naquira C, Ramirez E, et al. Research ethics training in Peru: a cases study. PLoS One 2008;3(9):e3274.

5. Saver R. What IRBs could learn from corporate boards. IRB. 2005;27(5):1-6.

6. World Health Organization. Surveying and evaluating ethical review practices: a complementary guideline to the operational guidelines for ethics committees that review biomedical research. Geneva: WHO; 2002.

7. Saunders C, Shefrin A, Rusnak E, DeMarinis AJ. Policies and standard operating procedures for the institutional review board. $2^{\text {nd }}$ ed. Boston: CenterWatch; 2008.

8. Conferencia Internacional de Armonización (CIARM). Guía Tripartita Armonizada de la Conferencia Internacional de Armonización. Lineamientos para la Buena Práctica Clínica (BPC) sobre requerimientos técnicos para el registro de productos farmacéuticos para uso en humanos. Ginebra: CIARM; 1997.

9. Pickworth E. Should local research ethics committees monitor research they have approved? J Med Ethics. 2000;26(5):330-33.

10. Council for International Organizations of Medical Sciences (CIOMS). International ethical guidelines for biomedical research involving human subjects. Geneva: CIOMS; 2002.

11. US Office of Human Subjects Research. Code of Federal Regulations. Title 45 CFR Part 46. Public welfare: Protection of human subjects. Bethesda: OHSR; 2005.

12. Instituto Nacional de Salud. Reglamento de ensayos clínicos. Lima: INS; 2010.

13. Sotomayor MA. Regulación de la investigación biomédica en Chile. Acta Bioeth. 2008;14(1):79-89.

14. Administración Nacional de Medicamentos, Alimentos y Tecnología Médica. Disposición ANMAT 5330/97. Buenas prácticas de investigación en estudios de farmacología clínica. Buenos Aires: ANMAT; 1997.

15. European Forum for Good Clinical Practice. European guidelines for auditing independent ethics committees. Brussels: EFGCP; 2002.

16. European Forum for Good Clinical Practice. Guidelines and recommendations for European ethics committees. Brussels: EFGCP; 1997.

17. Minaya-Martinez G, Díaz-Sandoval R. Capacidad de evaluación de los ensayos clínicos por los comités de ética. Rev Soc Peru Med Interna. 2008;21(4):153-64.

18. International Organization of Standardization. ISO 9001:2000. Quality management systems. Geneva: ISO; 2000. 
19. Koshore N, Antel S, Sargent K. The value of site based quality assurance systems for clinical testing sites. Monitor. $2000 ;: 29-32$.

20. Whalen M, Khin-Maung-Gyi F. Human research protections and good clinical practice (GCP): the relationship among GCP, the auditing process, and institutional review boards. J GXP Compliance. 2003;7(2):45-48.

21. Mc Alister S. Compliance with GCP at the investigator site. Are the lessons being learnt? Good Clin Practice J. 2000;7(2):22-25.

22. Handshaw K. Results of Internal IRB audit. Res Health Care. 2002;6:1-2.

23. Edwards SJ, Kirchin S, Huxtable R. Research ethics committees and paternalism. J Med Ethics. 2004;30(1):88-91.

24. Diario Clarín. Revelan que pruebas de medicamentos en EEUU causan muertos y heridos. Diario Clarín. 2005; Nov 03; Sociedad.
25. Van Lier A. Liaising with ethics committees according to ICH GCP. Good Clin Pract J. 2000;7(1):14-18.

26. Whalen M, Khin-Maung-Gyi F, Goebel P. The professionalization of clinical research. A continuum of learning for investigators, IRB administrative staffs \& institutional human research protection authorities: one approach. Monitor. 2001;:27-30.

27. Bota A, Estévez A, Fernández L, Hernández M, Hevia A, Lara C, et al. Los comités de evaluación ética y científica de la investigación en seres humanos en los países latinoamericanos y el Caribe: una propuesta para su desarrollo. Santiago de Chile: Universidad de Chile; 2004.

Correspondencia: Estela Quiroz

Correo electrónico: esteliqm@yahoo.com

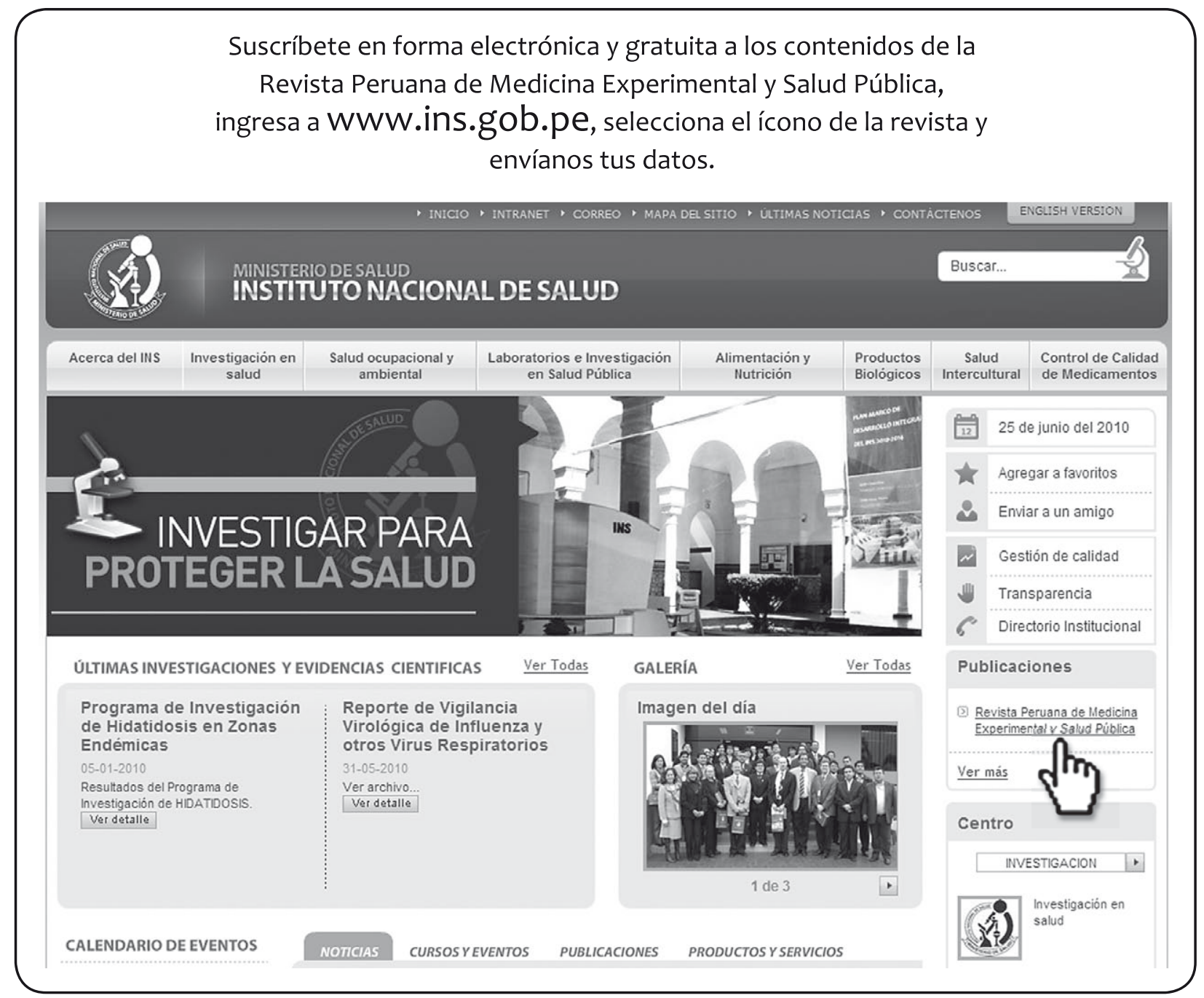

